delay treated at the Child Neuropsychiatry Unit, Department of Pediatrics, Second University of Naples, Italy. Over a median 7 month trial period, seizures were completely controlled in $22 \%$, and reduced by $>50 \%$ in $14 \%$. Absence and atonic seizures were controlled more effectively than partial or secondarily generalized seizures. Adverse effects occurred in $16 \%$, but treatment was discontinued only in 1 because of a skin rash. Some improvements in cognition, attention, and speech reported by parents and teachers were unrelated to decrease in seizure frequency. (Coppola G, Pascotto A. Lamotrigine as add-on drug in children and adolescents with refractory epilepsy and mental delay: an open trial. Brain Dev Sept 1997;19:398-402). (Respond: Dr Antonio Pascotto, Clinica di Neuropsichiatria Infantile, Dipartimento di Pediatria, Seconda Universita di Napoli, Via Pansini 5, 80131 Napoli, Italy).

COMMENT. Lamotrigine is an effective add-on therapy in children with refractory absence, atonic, and tonic-clonic seizures. It is relatively safe and free from serious side-effects. In this series, skin rash developed in 3 , but only one required drug withdrawal.

Lamotrigine in pregnancy and lactation was investigated in a patient at the Karolinska Institute and Hospital, Stockholm, Sweden. (Tomson T, Ohman I, Vitols S. Epilepsia Sept 1997;38:1039-1041). Plasma levels of lamotrigine (LTG) decreased as pregnancy progressed, suggesting enhanced clearance of LTG. LTG concentrations in the nursing infant (25\% of mother's plasma levels) were high due to passage of LTG into breast milk and slow elimination in the newborn.

\title{
TERATOGENIC EFFECTS OF ANTIEPILEPTIC DRUGS
}

Data from 5 prospective European studies totaling 1,221 children exposed to antiepileptic drugs (AED) during pregnancy and 158 children of unexposed control pregnancies were analyzed to quantify the risk of major congenital malformations (MCM) and are reported from University Medical Centers in Rotterdam and Heemstede, The Netherlands; Berlin and Magdeburg, Germany; and Helsinki, Finland. A comparison of a subgroup of 192 children exposed and 158 children unexposed to AED showed an increased risk of MCM (relative risk, RR 2.3 [1.2-4.7]). In those exposed to valproate or carbamazepine monotherapy, the RR was 4.9. Comparing polytherapy exposures in all 1,221 pregnancies, risks of MCM were significantly increased for phenobarbital/ethosuximide combination (RR 9.8), and the combination of PHT, PB, CBZ, and VPA (RR 11). VPA risks were dose related, especially for neural tube defects: offspring exposed to a maternal dose of $>1000 \mathrm{mg} /$ day (RR 6.8) were affected more often than $<600 \mathrm{mg}$ VPA/day exposures. VPA and CBZ were consistently associated with an increased risk of MCM. (Samren EB, van Duijn CM, Koch S et al. Maternal use of antiepileptic drugs and the risk of major congenital malformations: a joint European prospective study of human teratogenesis associated with maternal epilepsy. Epilepsia Sept 1997;38:981-990). (Reprints: Professor D Lindhout, Dept of Clinical Genetics, Erasmus University Rotterdam, PO Box 1738, NL-3000 DR Rotterdam, The Netherlands).

COMMENT. In a prospective multicenter study with pooled data, valproate and carbamazepine were especially teratogenic. In particular, the offspring of women receiving $>1000 \mathrm{mg} /$ day of VPA were at increased risk of having major congenital malformations. The authors suggest that VPA should be avoided during pregnancy when possible. In an editorial comment, Yerby MS cautions regarding conclusions derived from pooled data and multiple pregnancies. 